\title{
Special Issue: Blockchain Theories and Applications
}

\author{
Yimin Lin
}

Received: 9 November 2020 / Accepted: 28 November 2020 / Published online: 15 December 2020

(C) The Author(s), under exclusive licence to Springer Nature B.V. part of Springer Nature 2020

The emergence of blockchain for asset transfer without intermediaries has enabled a booming of systems and applications using novel technology. The primary pushing power for such systems comes from application domains which not only require trustless exchanges in decentralized environments but also desire data integrity, data immutability, data security, and privacy preservation. The increasing acceptance of blockchain technology as a mainstream system development framework suggests that the blockchain application areas can be broad.

The possibilities are tremendous, however, the adaptation of classical applications to blockchain technology is not straightforward and requires certain fundamental shifts in computing, storage, communication, finance, and other system requirements. And the challenges remain huge. In this special issue, several interesting and important areas together with related challenges are being explored. For instance, (1) OP Return transactions published on Bitcoin, are being analyzed to investigate the underline publishing patterns and behaviors; (2) a blockchain-based security architecture in named data networking (NDN)-powered vehicle networks for key management, cache poisoning remediation, and access control; (3) a blockchain-enabled cyber-security framework for industrial IoT based on key generation mechanisms over long-range and low-power wireless networks for fast encrypted data processing and transmission; (4) AirCargoChain, which enables direct

Y. Lin $(\bowtie)$

Lee Kong Chian School of Business, Singapore Management University, Singapore, Singapore

e-mail: ymlin@smu.edu.sg data sharing of deliveries, and with a highly scalable storage scheme.

The applicability of blockchain technology in the aforementioned areas is although exciting but not obvious and trivial as blockchain systems are still lagging in the aspects such as fast query processing, high throughput, efficient communication mechanism, and storage requirements, among others. Therefore, novel theories, technologies, and analysis methodologies are desired to address the limitations of blockchain technology in addition to reaping its benefits. The solutions proposed in this special issue are good efforts to this.

The whole peer-review process put great emphasis on ensuring a high quality of accepted contributions. Each paper was reviewed by at least two Program Committee (PC) members, and accepted after careful revisions according to the comments in order to provide their exact formulations.

We would like to thank the PC members for helping out this special issue and putting together high-quality papers that provide an up-to-date overview on the area of blockchain challenges and opportunities. In addition, we also wish to thank all authors for submitting their work to this special issue.

We hope that this special issue of Journal of Grid Computing forms concreate steps in the path to a promising future of blockchain.

Publisher's Note Springer Nature remains neutral with regard to jurisdictional claims in published maps and institutional affiliations. 